\title{
Erratum to: Intelligent Technologies for Manipulation Tasks
}

Anton Aliceychik ${ }^{1}$, Igor Orlov ${ }^{1}$, Vladimir Pavlovsky ${ }^{1}$, Vladimir Smolin $^{1}$, Dmitry Gribkov $^{2}$, Alexey Podoprosvetov ${ }^{2}$, Elizaveta Stepanova ${ }^{2}$, Elizaveta Elagina ${ }^{3}$, Elena Efimova $^{3}$, and Marina Shishova ${ }^{3}$

\footnotetext{
${ }^{1}$ Keldysh Institute of Applied Mathematics of RAS, Moscow atooxa@gmail.com, i.orlov@keldysh.ru,

\section{Erratum to:}

\section{Chapter 3 in: A. Kravets et al. (Eds.)}

Creativity in Intelligent Technologies and Data Science, DOI: 10.1007/978-3-319-23766-4_3

In an older version of this paper the first name of the 5th author was erroneously spelt "Dmity". This has been corrected. 